\title{
Potential Game Approach for Spectrum Sharing in Distributed Cognitive Radio Networks
}

\author{
I Wayan MUSTIKA ${ }^{\dagger a}$, Student Member, Koji YAMAMOTO ${ }^{\dagger}$, Member, Hidekazu MURATA ${ }^{\dagger}$, Senior Member, \\ and Susumu YOSHIDA ${ }^{\dagger}$, Fellow
}

\begin{abstract}
SUMMARY In a spectrum sharing system, lower-priority users are allowed to spatially reuse the spectrum allocated to higher-priority users as long as they do not disrupt communications of the latter. Therefore, to improve spectrum utilization, an important requirement for the former users is to manage the interference and ensure that the latter users can maintain reliable communications. In the present paper, a game theoretic framework of joint channel selection and power allocation for spectrum sharing in distributed cognitive radio networks is proposed. First, a utility function that captures the cooperative behavior to manage the interference and the satisfaction level to improve the throughput of the lower-priority users is defined. Next, based on the defined utility function, the proposed framework can be formulated as a potential game; thus, it is guaranteed to converge to a Nash equilibrium when the best response dynamic is performed. Simulation results show the convergence of the proposed potential game and reveal that performance improvements in terms of network throughput of the lower-priority users and outage probability of the higher-priority users can be achieved by the introduction of an adaptive coefficient adjustment scheme in the proposed utility function at the expense of the convergence to the Nash equilibrium.

key words: spectrum sharing, interference management, cognitive radio, game theory, potential game
\end{abstract}

\section{Introduction}

In view of limits on the available frequency spectrum and the inefficiency in spectrum usage, there is a need for a new communication paradigm to exploit the existing wireless spectrum opportunistically [1]. A new paradigm that can be used to enhance the utilization of limited spectrum resources in wireless networks is spectrum sharing. Cognitive radio has the potential to enable the spectrum sharing by providing wireless devices with the capability to sense the surrounding environment and intelligently adjust their transmission parameters in order to increase the efficiency of spectrum utilization [2], [3].

Spectrum sharing in cognitive radio networks is generally modeled by considering the coexistence of a higherpriority system and a lower-priority system in the network. The former system can be characterized by a higher-priority user (HU) in a given frequency band, while the latter system is cognitive radio that is able to sense the available spectrum, detect the presence of the HUs, and evaluate the interference that is generated to the HU's receivers [4]. The main challenge in cognitive radio networks is how to construct the

Manuscript received May 1, 2010.

Manuscript revised July 28, 2010.

${ }^{\dagger}$ The authors are with the Graduate School of Informatics, Kyoto University, Kyoto-shi, 606-8501 Japan.

a) E-mail: contact-h22@hanase.kuee.kyoto-u.ac.jp

DOI: 10.1587/transcom.E93.B.3284 spectrum sharing schemes in which the lower-priority users (LUs) are allowed to spatially reuse the spectrum as long as they do not cause harmful interference to the HUs. Harmful interference refers to a situation where the interference is large enough to cause significant disruption in service or quality. Therefore, the HUs can operate effectively as long as the received signal-to-interference and noise power ratio (SINR) exceeds an established threshold or the interference is less than the maximum allowable level.

Recently, game theory has been used to analyze the behavior of the cognitive radio network since such a network can be modeled as an interaction among individual rational nodes through radio environment. Game-theoretic approaches have been extensively used for distributed power control [5]-[7], interference avoidance [8], access control [9], [10], and distributed resource management in wireless networks [11], [12]. Nie et al. studied adaptive channel selection and power allocation in cognitive radio networks [12]. However, the higher-priority system was not taken into consideration, and therefore, it was not explicitly protected from interference due to spectrum access of the lowerpriority system. In addition, Bloem et al. proposed a Stackelberg game approach for power control and channel allocation in cognitive radio networks [7]. In their work, it was assumed that there was only one $\mathrm{HU}$ in the network and that the HU charged players a virtual price to use its licensed frequency band.

In this paper, joint channel selection and power allocation for spectrum sharing in distributed cognitive radio networks are formulated as a potential game by extending the work in [12]. We first propose a utility function that captures the cooperative behavior to manage the interference and the satisfaction level to improve the throughput by increasing the transmit power of LUs [13]. A potential function that reflects the change in the utility function of a deviating player is formulated. Thus, when the players change their action sequentially by following the best response dynamics, convergence to a Nash equilibrium is achieved in the proposed potential game. The best response dynamics represents the best action of a player in response to the other players' action. Second, we investigate the performance trade-off between the higher-priority system and the lower-priority system for specific values of certain parameters. We then propose an adaptive adjustment of the coefficient in the defined utility function for the purpose of adaptively controlling the interference based on the quality 
of service (QoS) constraints specified by the HUs.

The remainder of this paper is organized as follows. In Sect. 2, a system model for spectrum sharing is described. A game-theoretic framework for spectrum sharing is presented in Sect. 3 along with potential game formulation. Section 4 describes proposed spectrum sharing schemes based on the potential game approach. In Sect. 5, we investigate the convergence properties of the proposed potential game and analyze the performance of both the higher-priority system and the lower-priority system. Finally, we present the conclusion in Sect. 6.

\section{System Model for Spectrum Sharing}

We consider a cognitive radio network that consists of $N$ stationary transmitter-receiver pairs of LUs and $M$ transmitterreceiver pairs of HUs, uniformly distributed in a square area. One channel $c_{h}^{\mathrm{H}}$ from the set of channels $C$ is allocated to the HU transmitter $h \in \mathcal{M}=\{1, \ldots, M\}$. For ease of analysis of the interference protection for the HUs in each spectrum band, we assume that there is only one HU operates on each frequency channel. An LU transmitter $i \in \mathcal{N}=\{1, \ldots, N\}$ distributively selects a channel $c_{i}$ from the set of channels $C$ and a transmit power level $p_{i}$ from the set of transmit powers $\mathcal{P}$ to communicate with the intended receiver without causing harmful interference to the HUs. The combination of the selected channel and transmit power at the LU transmitter $i$ is denoted by the composite action $a_{i}=\left(c_{i}, p_{i}\right)$.

Let $p_{h}^{\mathrm{H}}$ denote the transmit power of HU transmitter $h$, so that the received signal-to-interference power and noise ratio (SINR) at the HU receiver $h$ can be expressed as

$$
\gamma_{h}^{\mathrm{H}}=\frac{G_{h h}^{\mathrm{HH}} p_{h}^{\mathrm{H}}}{\sum_{i=1}^{N} G_{h i}^{\mathrm{HL}} p_{i} \delta_{c_{h}^{\mathrm{H}} c_{i}}+n_{0}},
$$

where $G_{h h}^{\mathrm{HH}}$ is the link gain between the HU transmitter $h$ and $\mathrm{HU}$ receiver $h, G_{h i}^{\mathrm{HL}}$ is the link gain between the LU transmitter $i$ and HU receiver $h$, and $n_{0}$ denotes the noise power at each receiver.

We consider simultaneous transmission among users in both the higher-priority system and the lower-priority system where the HU keeps the orthogonality each other by transmitting on different frequency channels, while the LUs spatially reuse the channel that is allocated to the HU. Thus, the SINR measured at the LU receiver $i$ can be expressed as

$$
\gamma_{i}=\frac{G_{i i} p_{i}}{\sum_{j=1, j \neq i}^{N} G_{i j} p_{j} \delta_{c_{i} c_{j}}+\sum_{h=1}^{M} G_{i h}^{\mathrm{LH}} p_{h}^{\mathrm{H}} \delta_{c_{i} c_{h}^{\mathrm{H}}}+n_{0}},
$$

where $p_{i}$ denotes the transmit power of the LU transmitter $i$, $G_{i j}$ is the link gain between the LU transmitter $j$ and LU receiver $i, G_{i h}^{\mathrm{LH}}$ is the link gain between the $\mathrm{HU}$ transmitter $h$ and $\mathrm{LU}$ receiver $i, c_{h}^{\mathrm{H}}$ denotes the channel that is allocated to the HU pair $h$, and $\delta_{c_{i} c_{j}}$ is the interference function that indicates whether or not channel $c_{i}$ and channel $c_{j}$ are the same:

$$
\delta_{c_{i} c_{j}}= \begin{cases}1 & c_{i}=c_{j} \\ 0 & c_{i} \neq c_{j} .\end{cases}
$$

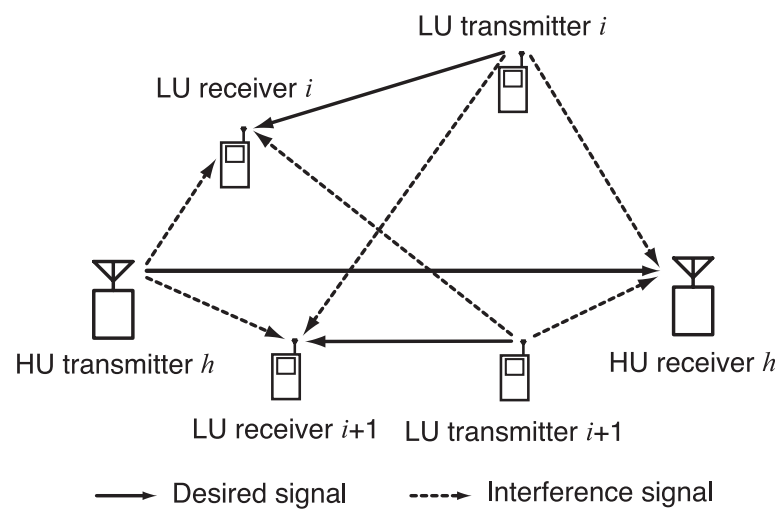

Fig. 1 System model.

The interference function $\delta_{c_{h}^{\mathrm{H}} c_{i}}$ and $\delta_{c_{i} c_{h}^{\mathrm{H}}}$ can also be derived using similar expression as $\delta_{c_{i} c_{j}}$.

Based on the received SINR at each LU receiver $i$, the average network throughput summed over all transmitterreceiver pairs of LUs can be estimated as

$$
T=\frac{1}{C} \sum_{i=1}^{N} \log _{2}\left(1+\gamma_{i}\right) .
$$

We refer the network throughput per unit bandwidth as the spectral efficiency, and it is assumed that the total available bandwidth is equally divided into $C$ available channels. Figure 1 shows an example of the spectrum sharing scenario in the cognitive radio network.

\section{Game-Theoretic Framework of Joint Channel Selec- tion and Power Allocation for Spectrum Sharing}

\subsection{Game-Theoretic Framework}

We formulate the joint channel selection and power allocation problem as a normal form game. A normal form game consists of a set of players, a strategy or action space for each player, and a set of utilities. It can be mathematically defined as $\Gamma=\left\{\mathcal{N},\left\{\mathcal{A}_{i}\right\}_{i \in \mathcal{N}},\left\{u_{i}\right\}_{i \in \mathcal{N}}\right\}$, where $\mathcal{N}$ is the finite set of players and $\mathcal{A}_{i}$ is the set of strategies or actions associated with player $i$. The strategy or action space of all players is $\mathcal{A}=\prod_{i \in \mathcal{N}} \mathcal{A}_{i}$ and the set of utility functions that associate players with their actions can be defined as $u_{i}: \mathcal{A} \rightarrow \mathbb{R}$, which indicates the mapping to a real value from each combination of the strategies of all players. In other word, the utility function of player $i$ can be represented as the degree of satisfaction of player $i$ as a function of the action it chooses, $a_{i}$, and the action of the other players, $\boldsymbol{a}_{-i}=\left(a_{1}, \ldots, a_{i-1}, a_{i+1}, \ldots, a_{N}\right)$.

As a fundamental concept of a normal form game, the Nash equilibrium helps to predict the outcome of the game. The Nash equilibrium can be defined as a point where no player can improve its utility by choosing another action, assuming that the other players' actions remain unchanged [14]. Let $a_{i}^{\star}$ denote the best strategy of player $i$ in response 
to the strategy of the other players. Then, a set of pure strategy profiles of all players, $\boldsymbol{a}^{\star}=\left(a_{i}^{\star}, \boldsymbol{a}_{-i}^{\star}\right) \in \mathcal{A}$, is a Nash equilibrium if and only if

$$
u_{i}\left(a_{i}^{\star}, \boldsymbol{a}_{-i}^{\star}\right) \geq u_{i}\left(a_{i}^{\prime}, \boldsymbol{a}_{-i}^{\star}\right), \forall a_{i}^{\prime} \neq a_{i}^{\star}, \forall a_{i}^{\prime} \in \mathcal{A}_{i}, \forall i \in \mathcal{N} .
$$

\subsection{Joint Channel Selection and Power Allocation for} Spectrum Sharing

We consider the set of LU pairs as players and the combination of channel and power as the set of actions. The objective of the game is to select a combination of channel and power for each LU pair in a distributed manner so as to maximize its utility function while ensuring that the QoS constraints (i.e., achieving the target SINR) of HUs are satisfied. Moreover, the set of HU pairs is not considered as players in our game. This is a reasonable assumption since, in practical situation, the HUs have exclusive rights to use the spectrum, but can allow the LUs to reuse the spectrum to improve the spectrum efficiency.

\subsection{Potential Game}

A certain class of games called potential games has been shown to always converge to a Nash equilibrium when only one player changes its action in each time step, following the best response dynamics [15]. The best response dynamics is a dynamic process of updating actions. In this process, a player chooses an action that maximizes its utility, given the current action of the other players [16]. The best response dynamics of player $i$ to the action profile $\boldsymbol{a}_{-i}$ at time $k+1$, $a_{i}^{(k+1)}\left(\boldsymbol{a}_{-i}\right)$, is an action that satisfies

$$
a_{i}^{(k+1)} \in \underset{a_{i}^{\prime} \in \mathcal{P}_{i}}{\arg \max } u_{i}\left(a_{i}^{\prime}, \boldsymbol{a}_{-i}^{k}\right),
$$

where $\left(a_{i}^{k}, \boldsymbol{a}_{-i}^{k}\right) \in \mathcal{A}$ denotes the action profile at time $k$.

A normal form game is called a potential game [15] if there exists a potential function $P: \mathcal{A} \rightarrow \mathbb{R}$ that satisfies

$$
P\left(a_{i}^{\prime}, \boldsymbol{a}_{-i}\right)-P\left(a_{i}, \boldsymbol{a}_{-i}\right)=u_{i}\left(a_{i}^{\prime}, \boldsymbol{a}_{-i}\right)-u_{i}\left(a_{i}, \boldsymbol{a}_{-i}\right),
$$

where $a_{i}^{\prime} \in \mathcal{A}_{i}$. The left-hand side of the above equation represents the change in the global objective function, called the potential function, while the right-hand side represents the change only in the utility of the deviating player $i$. The existence of a potential function that reflects the change in the utility function resulting from a unilateral deviation by the player is a characteristic of the potential game. Thus, when the player chooses the best action given the current action of the other players, the improvement in the utility of the player will lead to improvement in the value of potential function.

\section{Proposed Schemes}

\subsection{Proposed Utility and Potential Functions}

In [12], the authors proposed a utility function for cognitive radios by taking into account the interference power:

$$
u 1_{i}\left(a_{i}, \boldsymbol{a}_{-i}\right)=-\sum_{j=1, j \neq i}^{N} G_{j i} p_{i} \delta_{c_{j} c_{i}}-\sum_{j=1, j \neq i}^{N} G_{i j} p_{j} \delta_{c_{i} c_{j}} .
$$

The first and second terms in $u 1$ represent the interference power generated by transmitter $i$ and the total interference power perceived by receiver $i$, respectively.

The potential function of the above utility function is formulated as

$$
\begin{aligned}
& P 1\left(a_{i}, \boldsymbol{a}_{-i}\right) \\
& \quad=\sum_{i=1}^{N}\left(-\frac{1}{2} \sum_{j=1, j \neq i}^{N} G_{j i} p_{i} \delta_{c_{j} c_{i}}-\frac{1}{2} \sum_{j=1, j \neq i}^{N} G_{i j} p_{j} \delta_{c_{i} c_{j}}\right),
\end{aligned}
$$

where the weight factor $1 / 2$ is set so as to take into account the symmetric property of the interference power generated and the interference perceived by player $i$ [12].

We propose a utility function [13] that takes into account the interference power generated by the LU transmitter $i$ to the HU receiver $h$, the interference power received at the LU receiver $i$ from the HU transmitter $h$, and the satisfaction level of each LU to improve its throughput, in addition to the utility function $u 1$ proposed in [12]. The latter utility function is given as

$$
\begin{gathered}
u 2_{i}\left(a_{i}, \boldsymbol{a}_{-i}\right)=-\sum_{j=1, j \neq i}^{N} G_{j i} p_{i} \delta_{c_{j} c_{i}}-\sum_{j=1, j \neq i}^{N} G_{i j} p_{j} \delta_{c_{i} c_{j}} \\
-\alpha \sum_{h=1}^{M} G_{h i}^{\mathrm{HL}} p_{i} \delta_{c_{h}^{\mathrm{H}} c_{i}}-\sum_{h=1}^{M} G_{i h}^{\mathrm{LH}} p_{h}^{\mathrm{H}} \delta_{c_{i} \mathrm{C}_{h}^{\mathrm{H}}} \\
+\beta G_{i i} p_{i} .
\end{gathered}
$$

The first three terms capture the cooperative behavior to manage the interference created by the LU transmitters, the fourth term captures the total received interference power at an $\mathrm{LU}$ receiver from the $\mathrm{HU}$ transmitters, and the last term provides an incentive for each LU to improve its throughput by increasing the transmit power. In addition, the parameter $\alpha$ represents the importance of reducing the interference generated to the HU receiver by the LU transmitter, while $\beta$ represents the incentive to improve the throughput selfishly by each LU pair.

Given the proposed utility function $u 2$, we formulate the potential function as [13]

$$
\begin{aligned}
& P 2\left(a_{i}, \boldsymbol{a}_{-i}\right) \\
& =\sum_{i=1}^{N}\left(-\frac{1}{2} \sum_{j=1, j \neq i}^{N} G_{j i} p_{i} \delta_{c_{j} c_{i}}-\frac{1}{2} \sum_{j=1, j \neq i}^{N} G_{i j} p_{j} \delta_{c_{i} c_{j}}\right) \\
& \quad+\sum_{i=1}^{N}\left(-\alpha \sum_{h=1}^{M} G_{h i}^{\mathrm{HL}} p_{i} \delta_{c_{h}^{\mathrm{H}} c_{i}}-\sum_{h=1}^{M} G_{i h}^{\mathrm{LH}} p_{h}^{\mathrm{H}} \delta_{c_{i} \mathrm{C}_{h}^{\mathrm{H}}}\right) \\
& \quad+\sum_{i=1}^{N} \beta G_{i i} p_{i} .
\end{aligned}
$$


Because of the asymmetric property of the interference power generated by the LU transmitter $i$ to the $\mathrm{HU}$ receiver $h$ and the interference power received at LU receiver $i$ from the HU transmitter $h$, the weight factor $1 / 2$ is not applied to the third and fourth terms in the proposed $P 2$. The proof to show that the proposed $P 2$ is a potential function of $\left\{\mathcal{N},\left\{\mathcal{A}_{i}\right\}_{i \in \mathcal{N}},\left\{u 2_{i}\right\}_{i \in \mathcal{N}}\right\}$ is given in Appendix.

In addition, the proposed potential game requires that each LU transmitter has knowledge of the link gain between each transmitter-receiver pair of LUs and HUs and the transmit power of each LU transmitter and each HU transmitter, which can be obtained by exchanging control messages in the network, probing packets, and channel measurement.

4.2 Proposed Utility and Potential Functions with Adaptive Coefficient Adjustment for Spectrum Sharing

In the proposed scheme above, the parameter $\alpha$ is assumed to be fixed during the game, regardless of the action of each player. Intuitively, an increase in $\alpha$ represents an increase in the importance of reducing the interference generated to the $\mathrm{HU}$ receivers by the LU transmitters. Thus, as $\alpha$ increases, each LU transmitter tends to decrease its transmit power so as to maximize the utility function. As a result, it may degrade the throughput performance of the LUs. To adaptively control the interference based on the interference limit at each HU receiver, we propose a utility function with adaptive coefficient adjustment:

$$
\begin{gathered}
u 3_{i}\left(a_{i}, \boldsymbol{a}_{-i}\right)=-\sum_{j=1, j \neq i}^{N} G_{j i} p_{i} \delta_{c_{j} c_{i}}-\sum_{j=1, j \neq i}^{N} G_{i j} p_{j} \delta_{c_{i} c_{j}} \\
-\alpha_{i, c_{i}, p_{i}} \sum_{h=1}^{M} G_{h i}^{\mathrm{HL}} p_{i} \delta_{c_{h}^{\mathrm{H}} c_{i}}-\sum_{h=1}^{M} G_{i h}^{\mathrm{LH}} p_{h}^{\mathrm{H}} \delta_{c_{i} c_{h}^{\mathrm{H}}} \\
+\beta G_{i i} p_{i},
\end{gathered}
$$

where the main difference between the proposed utility functions $u 3$ and $u 2$ is in the third term.

In this scheme, the coefficient $\alpha_{i, c_{i}, p_{i}}$ is updated when player $i$ updates its action at time $k$ based on the following condition:

$$
\alpha_{i, c_{i}, p_{i}}[k]= \begin{cases}\alpha_{i, c_{i}, p_{i}}[k-1]-\Delta & I_{i, c_{i}, p_{i}}[k] \leq \frac{C \cdot I_{c_{i}}^{\text {limit }}}{N} \\ \alpha_{i, c_{i}, p_{i}}[k-1] & \text { otherwise }\end{cases}
$$

where $\alpha_{i, c_{i}, p_{i}}[0]=\alpha_{i, c_{i}, p_{i}}^{\max }, I_{i, c_{i}, p_{i}}[k]=\sum_{h=1}^{M} G_{h i}^{\mathrm{HL}} p_{i} \delta_{c_{h}^{\mathrm{H}} c_{i}}[k], C$ is the number of available channels, $N$ is the number of all LU pairs, and $I_{c_{i}}^{\text {limit }}$ denotes the interference limit of the HU receiver that operates on channel $c_{i} . \Delta$ is a non-negative step size, which is used to reduce the coefficient $\alpha_{i, c_{i}, p_{i}}$ and adjust the value of the third term in $u 3$. If the interference generated by the LU transmitter $i$ to the HU receiver $h$ is below the defined interference limit per user, $C \cdot I_{c_{i}}^{\text {limit }} / N$, the coefficient $\alpha_{i, c_{i}, p_{i}}$ will be decreased by the value of $\Delta$. By reducing $\alpha_{i, c_{i}, p_{i}}$ and adjusting the value of the third term in $u 3$, the LU transmitter $i$ can maximize its utility by increasing the transmit power $p_{i}$ due to less dominant of the third term in $u 3$. Since the coefficient $\alpha_{i, c_{i}, p_{i}}$ decreases once condition (13) is satisfied, we further consider $\alpha_{i, c_{i}, p_{i}} \geq 0$ to prevent $\alpha_{i, c_{i}, p_{i}}$ from becoming negative during the action update since it might discourage the LU transmitter from taking actions to avoid generating harmful interference to the HU receivers.

The motivation for the proposed adaptive coefficient adjustment in $u 3$ is to specify the weight in $\alpha_{i, c_{i}, p_{i}}$ based on the interference limit of the HU receiver that operates on each channel. During iteration time, the coefficient $\alpha_{i, c_{i}, p_{i}}$ is updated when the interference generated by the LU transmitter $i$ with a known transmit power $p_{i}$ to the $\mathrm{HU}$ receiver $h$ that operates on channel $c_{i}$ is lower or equal to the interference limit per user for that channel. Assuming that the players are equally distributed in all available channels, the interference limit per user for each channel, $C \cdot I_{c_{i}}^{\text {limit }} / N$, is considered to be an approximation of the maximum allowable interference that can be generated by each LU transmitter to the HU receiver in each channel.

In this scheme, we assume that each LU transmitter has knowledge of the number of all LU pairs and the interference limit at each HU receiver. Such information can be obtained from the exchange of control messages between the LUs and HUs in the network. However, the introduction of adaptive coefficient adjustment may increase the implementation complexity of $u 3$ since additional information is required to evaluate $u 3$, i.e., the target SINR at each HU receiver and the link gain between each transmitter-receiver pair of HU.

The potential function of $u 3$ can be formulated as

$$
\begin{aligned}
P 3\left(a_{i}, \boldsymbol{a}_{-i}\right) & \\
= & \sum_{i=1}^{N}\left(-\frac{1}{2} \sum_{j=1, j \neq i}^{N} G_{j i} p_{i} \delta_{c_{j} c_{i}}-\frac{1}{2} \sum_{j=1, j \neq i}^{N} G_{i j} p_{j} \delta_{c_{i} c_{j}}\right) \\
& +\sum_{i=1}^{N}\left(-\alpha_{i, c_{i}, p_{i}} \sum_{h=1}^{M} G_{h i}^{\mathrm{HL}} p_{i} \delta_{c_{h}^{\mathrm{H}} c_{i}}-\sum_{h=1}^{M} G_{i h}^{\mathrm{LH}} p_{h}^{\mathrm{H}} \delta_{c_{i} c_{h}^{\mathrm{H}}}\right) \\
& +\sum_{i=1}^{N} \beta G_{i i} p_{i} .
\end{aligned}
$$

Since the coefficient of $\alpha_{i, c_{i}, p_{i}}$ depends solely on the action of player $i, P 3$ can be shown to be a potential function.

\section{Simulation Results and Performance Comparisons}

In this section, we evaluate the performance of our proposed schemes by assuming specific values for certain parameters. We assume $N=30$ stationary transmitter-receiver pairs of LUs that are randomly distributed over a $400 \mathrm{~m} \times 400 \mathrm{~m}$ square area and the distance between the transmitter and receiver pair of LUs is less than $30 \mathrm{~m}$. With respect to strategy space, there are four channels with equal bandwidth and the set of transmit power levels is defined as $\mathcal{P}=\{1,2.5,5,10,15\} \mathrm{mW}$. Each LU transmitter selects the channel randomly and transmit power at $1 \mathrm{~mW}$ as the initial 


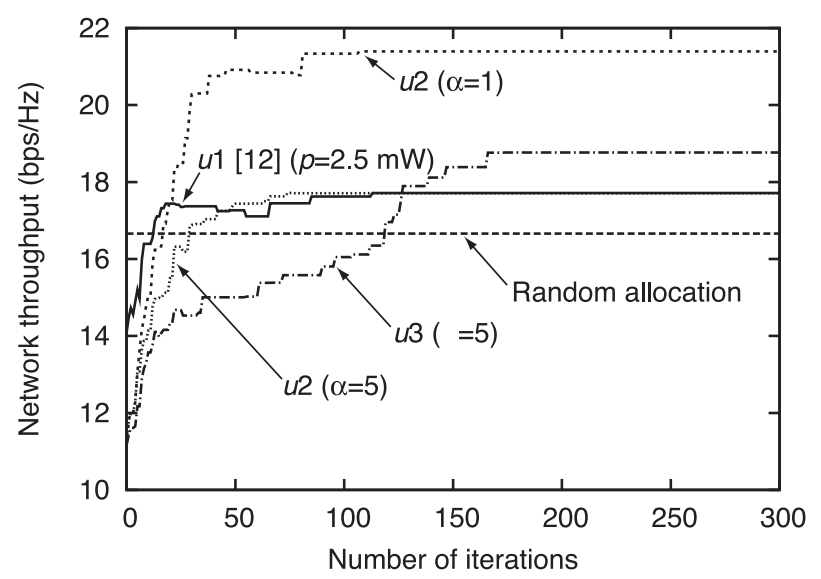

Fig. 2 Convergence of network throughput for LUs.

Table 1 Action of the LU pair in each scheme.

\begin{tabular}{c|c|c}
\hline Scheme & Channel & Power \\
\hline Random allocation & $\times$ & $\times$ \\
$u 1[12]$ & $\bigcirc$ & $\times$ \\
$u 2$ & $\bigcirc$ & $\bigcirc$ \\
$u 3$ & $\bigcirc$ & $\bigcirc$ \\
\hline
\end{tabular}

assignment. Unless otherwise specified, we assume $M=4$ stationary transmitter-receiver pairs of HUs where each pair of HUs operates on each frequency channel with transmission power at $25 \mathrm{~mW}$; the maximum distance between the transmitter and receiver pair of HUs is $30 \mathrm{~m}$. The simulation is carried out for 5000 different topologies.

To illustrate the QoS constraints of the higher-priority system, we assume that each HU receiver has a minimum required SINR, denoted as $\gamma_{h}^{\text {target }}$. Thus, the interference limit of the HU receiver that operates on channel $c_{i}$, denoted as $I_{c_{i}}^{\text {limit }}$, can be estimated by each LU transmitter $i$ in $u 3$. Unless otherwise specified, $\gamma_{h}^{\text {target }}$ is set to $15 \mathrm{~dB}$. We consider the free space path loss propagation model with path loss exponent of 2. Each LU and each HU are equipped with an omnidirectional antenna with identical propagation characteristics and the noise power $n_{0}$ at each receiver is set to $10^{-13} \mathrm{~W}$. For the sake of simplicity, the effect of fast fading and shadowing are not considered in the simulation. Furthermore, we consider the simultaneous transmission of all transmitter-receiver pairs of LUs over a shared spectrum and each transmitter is assumed to transmit packets at all times.

\subsection{Convergence Properties of Joint Channel Selection and Power Allocation Schemes}

Figure 2 shows the evolution of the network throughput of each scheme during the iteration time when the players update their action sequentially. In the random allocation scheme, channel and power are allocated randomly to each LU transmitter during the initial transmission and they remain unchanged during the iteration time. Therefore, the network throughput of the random allocation scheme remains unchanged during the iteration time. For $u 1$, we con-

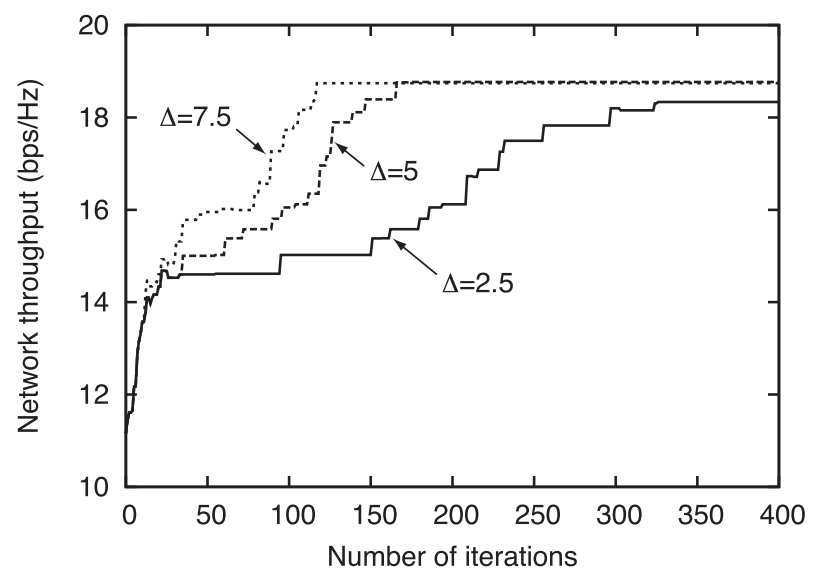

Fig. 3 Impact of parameter $\Delta$ to the convergence speed of $u 3$.

sider channel selection with a fixed transmit power, which is one of the schemes proposed in [12]. The action that can be selected by the LU pair in each scheme is summarized in Table 1. In each scheme, HU pairs are not considered as players, and therefore they do neither change the channel nor the transmission power. For both proposed $u 2$ and $u 3, \beta$ is set to 0.1 . Unless otherwise specified, $\Delta$ is set to 5 and $\alpha_{i, c_{i}, p_{i}}^{\min }$ and $\alpha_{i, c_{i}, p_{i}}^{\max }$ are set to 1 and 25, respectively, in $u 3$. Since $\Delta$ is the step size in the adaptive coefficient adjustment scheme, it affects the convergence speed of $u 3$ as shown in Fig. 3. The lower the value of $\Delta$, the slower the convergence to a Nash equilibrium. We also see from Fig. 3 that different values of $\Delta$ do not converge to the same Nash equilibrium. It is because the combination of strategies of all LU pairs at the Nash equilibrium point may be different under different value of $\Delta$ in $u 3$. From Fig. 2, we see that the best response in the case of $u 2$ and $u 3$ converges to a Nash equilibrium, which is represented by the steady-state condition of the network throughput of LUs. It also shows that the introduction of the adaptive coefficient adjustment in $u 3$ leads to slower convergence to a Nash equilibrium.

Figures 4 and 5 show that the best response in the case of $u 3$ leads to the updating of the channel and power of all players. During the iteration time, the LU pair may switch from one channel to another, which is shown by the transition of vertical lines in Fig. 4. Moreover, Fig. 5 shows the power selection of some LU pairs during the iteration time. By distributed sequential play and following the best response dynamics, each LU pair tries to maximize its utility by choosing the best action (channel and power) in response to the action of the other LU pairs. After some iteration steps, all LU pairs reach the convergence point, from which no LU pair would deviate anymore from its action.

\subsection{Effect of Parameters on the Performance of the Pro- posed Scheme}

Since the performance of $u 2$ depends on the parameters $\alpha$ and $\beta$, it is necessary to determine their appropriate values to satisfy a certain transmission probability of the HUs. As 


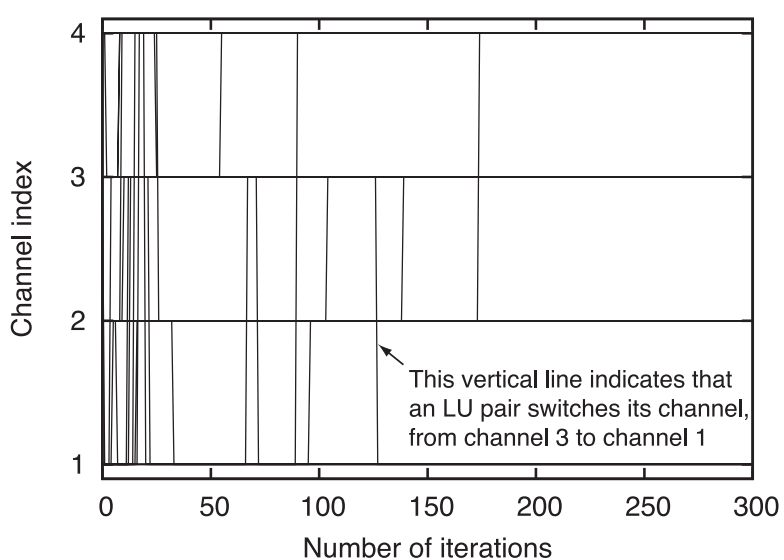

Fig. 4 Channel selection in $u 3$ ( $\Delta=5$ and $\beta=0.1$ ).

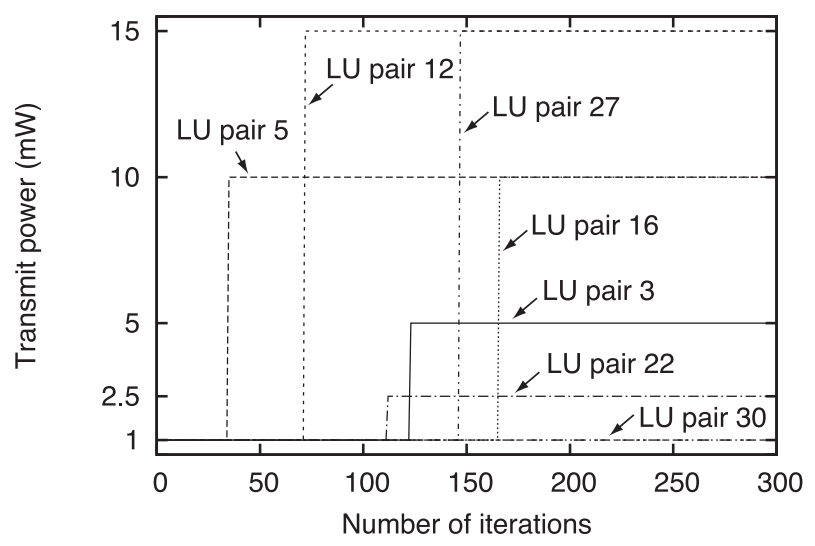

Fig. 5 Power allocation in $u 3(\Delta=5$ and $\beta=0.1)$.

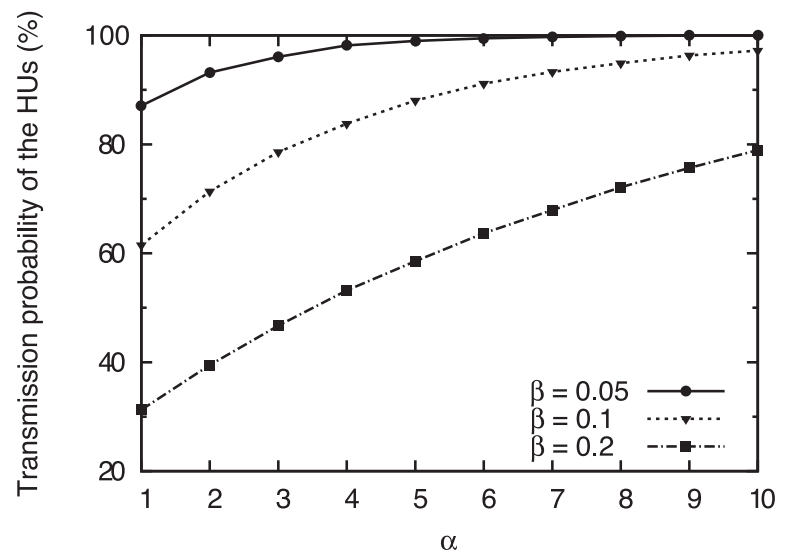

Fig. 6 Transmission probability of HUs vs. $\alpha$ in $u 2$.

we see in Fig. 6, the transmission probability of HUs increases with $\alpha$. One of the reason is that the third term of $u 2$ dominates the other terms in the case where $\alpha$ increases while $\beta$ remains fixed. Thus, each LU pair tries to maximize $u 2$ by selecting a low-interference channel and decreasing the transmit power. As the aggregate interference at $\mathrm{HU}$ receivers is reduced, the transmission probability of HUs can be increased.

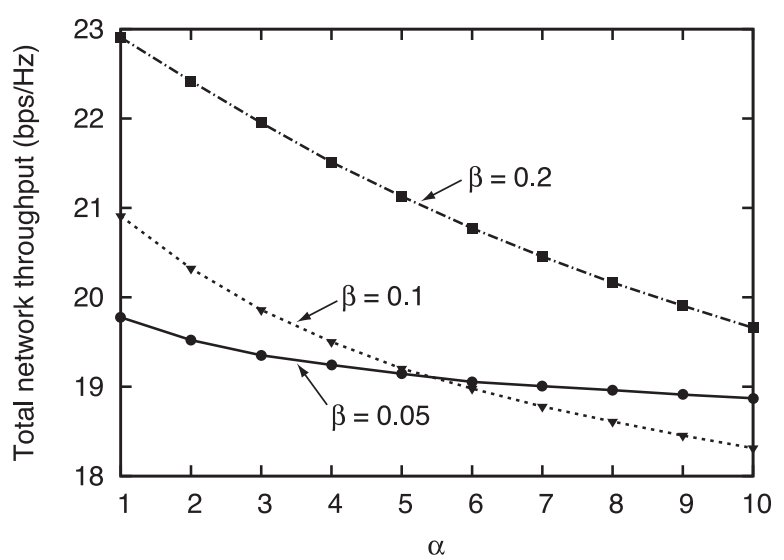

Fig. 7 Network throughput of LUs vs. $\alpha$ in $u 2$.

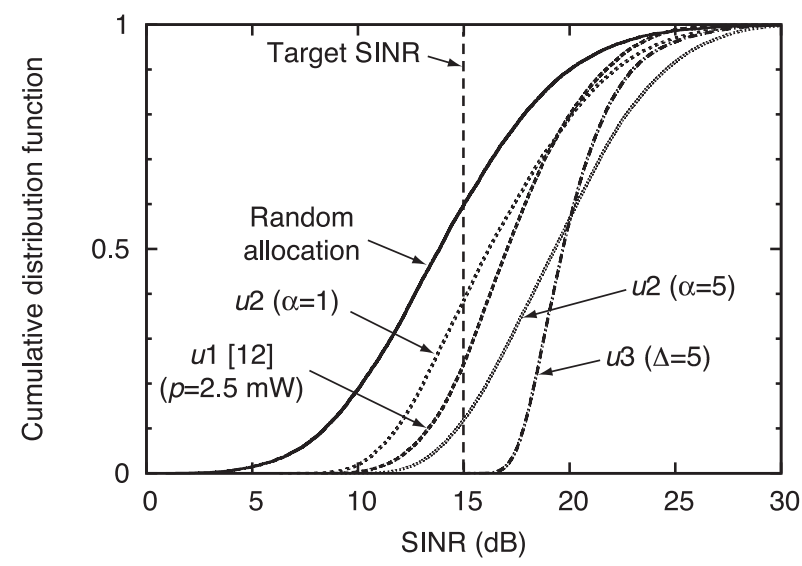

Fig. 8 Cumulative distribution function of the received SINR at HU pairs separated by different distances.

The relationship between the total network throughput of LUs and $\alpha$ for specific values of $\beta$ is shown in Fig. 7. As $\alpha$ increases, the total network throughput of LUs decreases due to reduced incentive to increase the transmit power for each LU. In particular, the higher the value of $\beta$, the more is the number of LU pairs that are encouraged to improve the throughput selfishly by increasing their transmit power at the expense of lower transmission probability of HUs, as shown in Fig. 6. This condition is considered as a performance trade-off between the higher-priority system and the lower-priority system.

\subsection{Performance Comparisons}

The cumulative distribution function (CDF) of the received SINR at HUs and the total network throughput of LUs averaged over 5000 different topologies with random distances and a fixed distance between HUs in a pair are shown in Figs. $8-10$. Note that the target SINR at each HU receiver is set to $15 \mathrm{~dB}$, and thus, the interference limit at each $\mathrm{HU}$ receiver may vary in the case of random distances. Figures 810 show the performance trade-off between the probability of the received SINR at HUs that exceeds the target SINR 


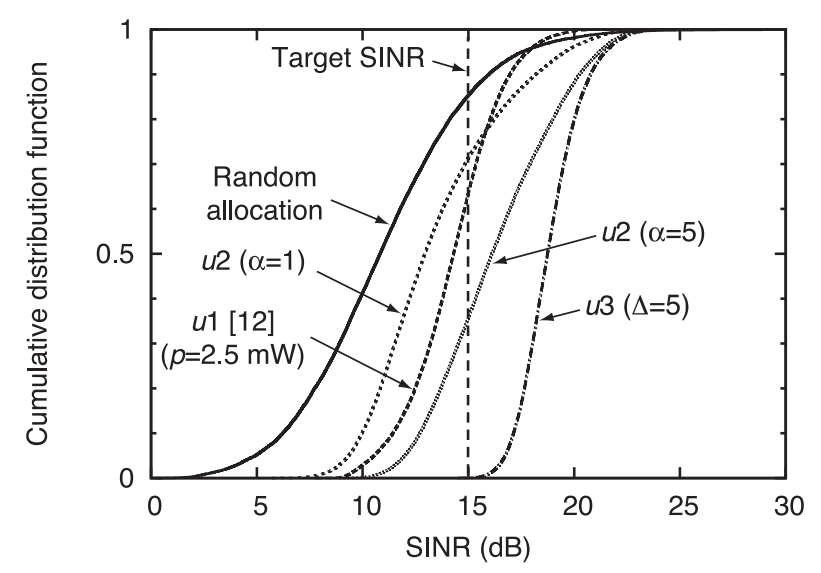

Fig. 9 Cumulative distribution function of the received SINR at HU pairs separated by a fixed distance $(d=30 \mathrm{~m})$.

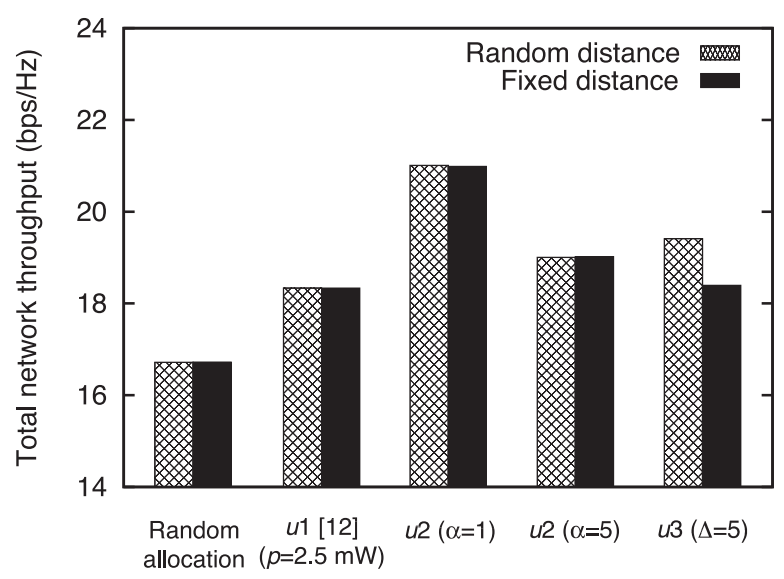

Fig. 10 Total average network throughput of LUs.

(transmission probability) and the total network throughput of LUs in $u 2$ with $\alpha=1$ and $\alpha=5$, which is also confirmed in Figs. 6 and 7.

The improvements in the performance of $u 3$ with adaptive coefficient adjustment are shown in Figs. 8-10. We see from Figs. 8 and 9 that $u 3$ achieves better interference management of the lower priority system in the coexistence of the higher priority system by adaptively controlling the interference from the LU transmitter to the $\mathrm{HU}$ receiver based on the target SINR at each HU receiver, while maintaining the network throughput of LUs does not degrade as much as that in $u 2(\alpha=5)$ as shown in Fig. 10. However, in the fixed distance scenario, there is a significant degradation of the network throughput of LUs in $u 3$ due to more stringent interference limit at each $\mathrm{HU}$ receiver.

From Figs. 8 and 9, we see that $u 2$ cannot satisfy the QoS requirement of HUs at the given target SINR $15 \mathrm{~dB}$ when $\alpha$ is set to either 1 or 5 under $\beta=0.1$. Therefore, in order to guarantee the QoS requirement of HUs, we have to set $\alpha \geq 10$ under $\beta=0.05$ in $u 2$ as shown in Fig. 6 . However, as the trade-off performance between the higherpriority system and the lower-priority system, the degradation of network throughput of LUs should be relaxed for

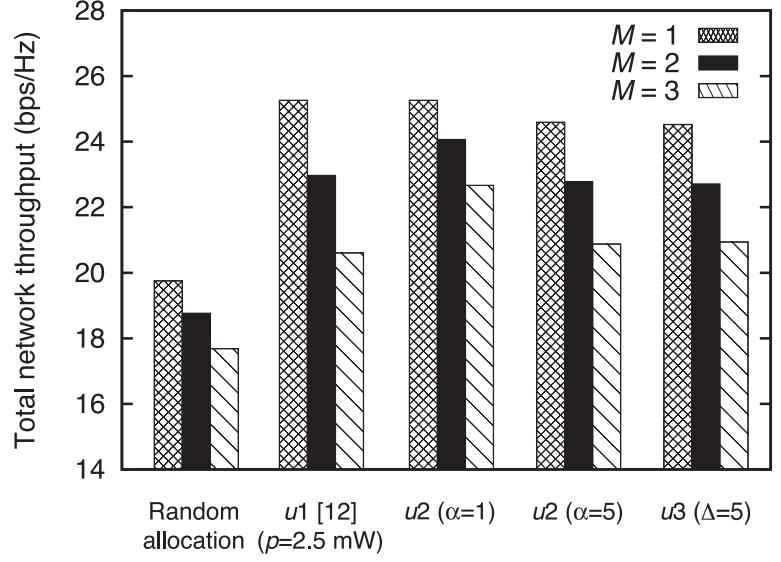

Fig. 11 Total average network throughput of LUs for the case where $M<C$.

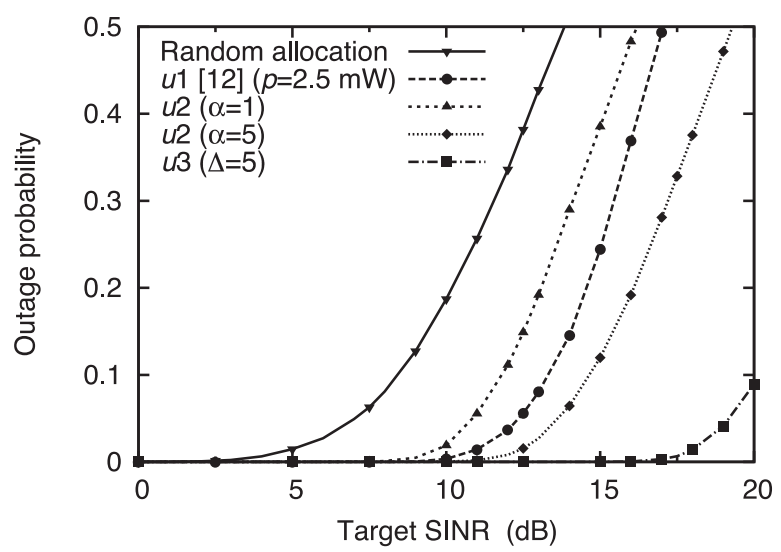

Fig. 12 Outage probability at HUs for different target SINRs $(\beta=0.1$ in $u 2$ and $u 3$ ).

the sake of guaranteeing the QoS requirement of HUs in $u 2$. Meanwhile, $u 3$ with adaptive coefficient adjustment guarantees the QoS requirement of HUs by maintaining the received SINR higher than the target SINR at HU receivers.

Figure 11 shows the network throughput of LUs for the case where the number of HU pairs is less than the number of available channels $(M<C)$. We see from Fig. 11 that reducing the number of $\mathrm{HU}$ pairs leads to the improvement of the network throughput of LUs in $u 2$ and $u 3$. In the case where $M=1$, there is no significant difference in the network throughput of LUs between $u 2$ and $u 3$. The reason is that most LU pairs prefer to choose the channels that are not used by the $\mathrm{HU}$ pair to avoid the interference from the $\mathrm{HU}$ transmitter to the LU receiver. As a result, the effect of the proposed schemes is limited due to less influence from the third and fourth terms in $u 2$ and $u 3$.

Figure 12 shows the outage probability of HU receivers under different target SINRs; the outage probability is defined as the probability of the received SINR at HU receivers being lower than the target SINR. From Fig. 12, we see that at the target SINR above $10 \mathrm{~dB}$, the outage probability of both $u 1[12]$ and $u 2$ increases as the target SINR increases. 
With the advantage of the feedback information from HUs, $u 3$ with adaptive coefficient adjustment achieves lower outage probability compared to that of the other schemes.

\section{Conclusion}

In this paper, we have proposed the potential game approach of joint channel selection and power allocation for spectrum sharing in distributive cognitive radio networks. In the proposed spectrum sharing schemes, the lower-priority users spatially reuse the spectrum and manage interference by defining a utility function, which can be formulated as a potential game. We demonstrate that the proposed potential game is guaranteed to converge to a Nash equilibrium when the players change their action sequentially, following the best response dynamics. The simulation results show that the proposed utility function with adaptive coefficient adjustment can achieve better performance improvements in terms of the network throughput of the lower-priority users and the outage probability of the higher-priority users compared to a utility function without coefficient adjustment, at the expense of slower convergence to a Nash equilibrium and an increase in the control overhead.

We would like to emphasize that the purpose of this paper is to present the interference management framework for spectrum sharing in cognitive radio networks using the potential game approach. Further research is required to investigate its applicability to more realistic models and to develop an efficient technique to reduce the information exchange in the network.

\section{Acknowledgments}

This work is supported in part by a Grant-in-Aid for Scientific Research (A) (no. 20246067) from the Japan Society for the Promotion of Science (JSPS). The first author would like to thank the Japan International Cooperation Agency (JICA) through AUN/SEED-Net project for supporting the study at Kyoto University.

\section{References}

[1] Q. Zhao and B.M. Sadler, "A survey of dynamic spectrum access: Signal processing, networking, and regulatory policy," IEEE Signal Process. Mag., vol.24, no.3, pp.79-89, May 2007.

[2] J. Mitola, "Cognitive radio: An integrated agent architecture for software defined radio," Doctor of Technology Dissertation, Royal Institute of Technology (KTH), Stockholm, Sweden, 2000.

[3] I.F. Akyildiz, W.-Y. Lee, M.C. Vuran, and S. Mohanty, "A survey on spectrum management in cognitive radio networks," IEEE Commun. Mag., vol.46, no.4, pp.40-48, April 2008.

[4] S. Haykin, "Cognitive radio: Brain-empowered wireless communications," IEEE J. Sel. Areas Commun., vol.23, no.2, pp.201-220, Feb. 2005.

[5] C.U. Saraydar, N.B. Mandayam, and D.J. Goodman, "Efficient power control via pricing in wireless data networks," IEEE Trans. Commun., vol.50, no.2, pp.291-303, Feb. 2002.

[6] T. Heikkinen, "A potential game approach to distributive power control and scheduling," Elsevier Computer Networks, vol.50, pp.22952311, Sept. 2006.
[7] M. Bloem, T. Alpcan, and T. Basar, "A stackelberg game for power control and channel allocation in cognitive radio networks," Proc. GameComm'07, Oct. 2007.

[8] R. Menon, A.B. MacKenzie, R.M. Buehrer, and J.H. Reed, "Interference avoidance in networks with distributed receivers," IEEE Trans. Commun., vol.57, no.10, pp.3078-3091, Oct. 2009.

[9] T. Cui, L. Chen, and S.H. Low, "A game-theoretic framework for medium access control,” IEEE J. Sel. Areas Commun., vol.26, no.7, pp.1116-1127, Sept. 2008.

[10] Y. Xiao, X. Shan, and Y. Ren, "Game theory models for IEEE 802.11 DCF in wireless ad hoc networks," IEEE Radio Commun. Mag., vol.43, no.3, pp.S22-S26, March 2005.

[11] E. Altman, T. Boulogne, R. El-Azouzi, T. Jimenez, and L. Wynter, "A survey on networking games in telecommunications," Computers and Operations Research, vol.33, pp.286-311, Feb. 2006.

[12] N. Nie, C. Comaniciu, and P. Agrawal, "A game theoretic approach to interference management in cognitive networks," in Wireless Communications (The IMA Volumes of Mathematics and its Applications), Springer, Nov. 2006.

[13] I.W. Mustika, K. Yamamoto, H. Murata, and S. Yoshida, "Spectrum sharing with interference management for distributed cognitive radio networks: A potential game approach,' Proc. IEEE VTC'10-Spring, May 2010

[14] D. Fudenberg and J. Tirole, Game Theory, MIT Press, 1991.

[15] D. Monderer and L.S. Shapley, "Potential games," J. Games and Economic Behavior, vol.14, pp.124-143, May 1996

[16] M. Voorneveld, "Best-response potential games," Economic Letters, vol.66, pp.289-295, March 2000.

\section{Appendix: Proof of the Proposed Potential Function}

Here, we prove the validity of the potential function $P 2\left(a_{i}, \boldsymbol{a}_{-i}\right)$ defined in (11). First, the potential function $P 2\left(a_{i}, a_{-i}\right)$ can be decomposed into three parts as follows:

$$
P 2\left(a_{i}, \boldsymbol{a}_{-i}\right)=P 2^{(1)}\left(a_{i}, \boldsymbol{a}_{-i}\right)+P 2^{(2)}\left(a_{i}, \boldsymbol{a}_{-i}\right)+P 2^{(3)}\left(a_{i}, \boldsymbol{a}_{-i}\right),
$$

where

$$
\begin{aligned}
& P 2^{(1)}\left(a_{i}, \boldsymbol{a}_{-i}\right)=\sum_{i=1}^{N}\left(-\frac{1}{2} \sum_{j=1, j \neq i}^{N} G_{j i} p_{i} \delta_{c_{j} c_{i}}-\frac{1}{2} \sum_{j=1, j \neq i}^{N} G_{i j} p_{j} \delta_{c_{i} c_{j}}\right), \\
& P 2^{(2)}\left(a_{i}, \boldsymbol{a}_{-i}\right)=\sum_{i=1}^{N}\left(-\alpha \sum_{h=1}^{M} G_{h i}^{\mathrm{HL}} p_{i} \delta_{c_{h}^{\mathrm{H}} c_{i}}-\sum_{h=1}^{M} G_{i h}^{\mathrm{LH}} p_{h}^{\mathrm{H}} \delta_{c_{i} c_{h}^{\mathrm{H}}}\right), \\
& P 2^{(3)}\left(a_{i}, \boldsymbol{a}_{-i}\right)=\sum_{i=1}^{N} \beta G_{i i} p_{i} .
\end{aligned}
$$

Since $P 2^{(1)}\left(a_{i}, \boldsymbol{a}_{-i}\right)$ has been proved in [12], we omit the proof for this term.

$P 2^{(2)}\left(a_{i}, \boldsymbol{a}_{-i}\right)$ can be derived as

$$
\begin{aligned}
P 2^{(2)}\left(a_{i}, \boldsymbol{a}_{-i}\right)=-\alpha \sum_{h=1}^{M} G_{h i}^{\mathrm{HL}} p_{i} \delta_{c_{h}^{\mathrm{H}} c_{i}}-\sum_{h=1}^{M} G_{i h}^{\mathrm{LH}} p_{h}^{\mathrm{H}} \delta_{c_{i} c_{h}^{\mathrm{H}}} \\
+\sum_{k=1, k \neq i}^{N}\left(-\alpha \sum_{h=1}^{M} G_{h k}^{\mathrm{HL}} p_{k} \delta_{c_{h}^{\mathrm{H}} c_{k}}-\sum_{h=1}^{M} G_{k h}^{\mathrm{LH}} p_{h}^{\mathrm{H}} \delta_{c_{k} c_{h}^{\mathrm{H}}}\right) .
\end{aligned}
$$

Let

$$
P 2^{(2)}\left(\boldsymbol{a}_{-i}\right)=\sum_{k=1, k \neq i}^{N}\left(-\alpha \sum_{h=1}^{M} G_{h k}^{\mathrm{HL}} p_{k} \delta_{c_{h}^{\mathrm{H}} c_{k}}-\sum_{h=1}^{M} G_{k h}^{\mathrm{LH}} p_{h}^{\mathrm{H}} \delta_{c_{k} c_{h}^{\mathrm{H}}}\right) .
$$


Then $P 2^{(2)}\left(a_{i}, \boldsymbol{a}_{-i}\right)$ can be decomposed as

$$
\begin{aligned}
& P 2^{(2)}\left(a_{i}, \boldsymbol{a}_{-i}\right) \\
& \quad=-\alpha \sum_{h=1}^{M} G_{h i}^{\mathrm{HL}} p_{i} \delta_{c_{h}^{\mathrm{H}} c_{i}}-\sum_{h=1}^{M} G_{i h}^{\mathrm{LH}} p_{h}^{\mathrm{H}} \delta_{c_{i} c_{h}^{\mathrm{H}}}+P 2^{(2)}\left(\boldsymbol{a}_{-i}\right) .
\end{aligned}
$$

$P 2^{(3)}\left(a_{i}, \boldsymbol{a}_{-i}\right)$ can be derived as

$$
P 2^{(3)}\left(a_{i}, \boldsymbol{a}_{-i}\right)=\beta G_{i i} p_{i}+\sum_{k=1, k \neq i}^{N} \beta G_{k k} p_{k} .
$$

Let

$$
P 2^{(3)}\left(\boldsymbol{a}_{-i}\right)=\sum_{k=1, k \neq i}^{N} \beta G_{k k} p_{k} .
$$

Then $P 2^{(3)}\left(a_{i}, \boldsymbol{a}_{-i}\right)$ can be decomposed as

$$
P 2^{(3)}\left(a_{i}, \boldsymbol{a}_{-i}\right)=\beta G_{i i} p_{i}+P 2^{(3)}\left(\boldsymbol{a}_{-i}\right),
$$

where the action of player $i$ in $P 2^{(1)}\left(a_{i}\right), P 2^{(2)}\left(a_{i}\right)$, and $P 2^{(3)}\left(a_{i}\right)$ does not depend on the action of the other players, $\boldsymbol{a}_{-i}$. The function $Q\left(\boldsymbol{a}_{-i}\right)$ corresponding to $\boldsymbol{a}_{-i}$ can be expressed as

$$
Q\left(\boldsymbol{a}_{-i}\right)=P 2^{(1)}\left(\boldsymbol{a}_{-i}\right)+P 2^{(2)}\left(\boldsymbol{a}_{-i}\right)+P 2^{(3)}\left(\boldsymbol{a}_{-i}\right),
$$

and $Q\left(\boldsymbol{a}_{-i}\right)$ is not affected by the action of the deviating player $i$. If player $i$ changes its action from $a_{i}$ to $a_{i}^{\prime}$, then we obtain

$$
P 2\left(a_{i}^{\prime}, \boldsymbol{a}_{-i}\right)=u 2_{i}\left(a_{i}^{\prime}, \boldsymbol{a}_{-i}\right)+Q\left(\boldsymbol{a}_{-i}\right) .
$$

Consequently,

$$
\begin{aligned}
P 2 & \left(a_{i}^{\prime}, \boldsymbol{a}_{-i}\right)-P 2\left(a_{i}, \boldsymbol{a}_{-i}\right) \\
& =\left(u 2_{i}\left(a_{i}^{\prime}, \boldsymbol{a}_{-i}\right)+Q\left(\boldsymbol{a}_{-i}\right)\right)-\left(u 2_{i}\left(a_{i}, \boldsymbol{a}_{-i}\right)+Q\left(\boldsymbol{a}_{-i}\right)\right) \\
& =u 2_{i}\left(a_{i}^{\prime}, \boldsymbol{a}_{-i}\right)-u 2_{i}\left(a_{i}, \boldsymbol{a}_{-i}\right) .
\end{aligned}
$$

This proves that the game $\left\{\mathcal{N},\left\{\mathcal{A}_{i}\right\}_{i \in \mathcal{N}},\left\{u 2_{i}\right\}_{i \in \mathcal{N}}\right\}$ is a potential game with a potential function $P 2$.

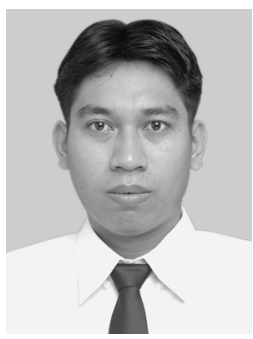

I Wayan Mustika was born in Denpasar, Indonesia, in 1981. He received the B.Eng. degree in Electrical Engineering from Gadjah Mada University, Indonesia, in 2005, and the M.Eng. degree in Computer Engineering from King Mongkut's Institute of Technology Ladkrabang (KMITL), Thailand, in 2008 with support of the Japan International Cooperation Agency (JICA) scholarship through AUN/Seed-Net project. He is currently a recipient of the JICA scholarship, studying towards his Ph.D. degree at the Graduate School of Informatics, Kyoto University. His research interests include routing protocol in mobile ad hoc networks and resource management in cognitive radio networks with a particular emphasis on spectrum sharing and game theory. He is a student member of the IEEE.

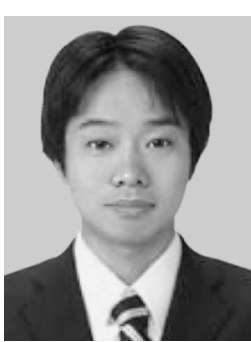

Koji Yamamoto received the B.E. degree in electrical and electronic engineering from Kyoto University in 2002, and the M.E. and Ph.D. degrees in informatics from Kyoto University in 2004 and 2005, respectively. From 2004-2005, he was a research fellow of the Japan Society for the Promotion of Science (JSPS). Since 2005, he has been an Assistant Professor of the Graduate School of Informatics, Kyoto University. From 2008 to 2009 , he was a visiting researcher at Wireless@KTH, Royal Institute of Technology (KTH), Sweden. His research interests include game theory, spectrum sharing, and cooperative multi-hop networks. He received the PIMRC 2004 Best Student Paper Award in 2004, the Ericsson Young Scientist Award in 2006, and the Young Researcher's Award from the IEICE of Japan in 2008. $\mathrm{He}$ is a member of the IEEE.

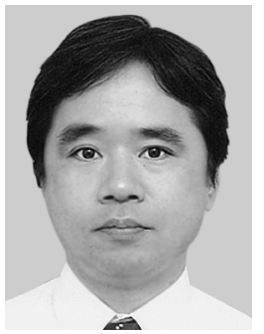

Hidekazu Murata received the B.S., M.E., and Ph.D. degrees in electronic engineering from Kyoto University, Kyoto, Japan, in 1991 , 1993, and 2000, respectively. In 1993, he joined the Faculty of Engineering, Kyoto University. From 2002 to 2006, he was an Associate Professor of Tokyo Institute of Technology. He has been at Kyoto University since October 2006 and is currently an Associate Professor of Department of Communications and Computer Engineering, Graduate School of Informatics. His major research interests include signal processing and its hardware implementation, with particular application to cooperative wireless networks with cognitive radio capabilities. He received the Young Researcher's Award from the IEICE of Japan in 1997, the Ericsson Young Scientist Award in 2000, and the Young Scientists' Prize of the Commendation for Science and Technology by the Minister of Education, Culture, Sports, Science and Technology in 2006. He is a member of the IEEE and SITA.

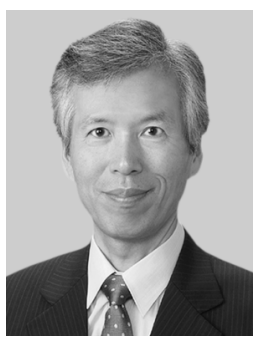

Susumu Yoshida received the B.E., M.E and $\mathrm{Ph} . \mathrm{D}$. degrees all in electrical engineering from Kyoto University, Kyoto, Japan in 1971, 1973 and 1978, respectively. Since 1973, he has been with the Faculty of Engineering, Kyoto University and currently he is a full professor of the Graduate School of Informatics, Kyoto University. During the last 30 years, he has been mainly engaged in the research of wireless personal communications. His current research interest includes highly spectrally efficient wireless transmission techniques and distributed controlled wireless networks. During 1990-1991, he was a visiting scholar at WINLAB, Rutgers University, U.S.A. and Carleton University in Ottawa, Canada. He served as a TPC Chair of IEEE VTC 2000-Spring, Tokyo. He was a guest editor of IEEE J-SAC on Wireless Local Communications published in April and May 1996. He received the IEICE Achievement Award and Ericsson Telecommunication Award in 1993 and 2007, respectively. 